\title{
What Is the Role of Nanotechnology in Diagnosis and Treatment of Metastatic Breast Cancer? Promising Scenarios for the Near Future
}

\author{
Truffi Marta, ${ }^{1}$ Sorrentino Luca, ${ }^{1,2}$ Mazzucchelli Serena, ${ }^{1}$ Fiandra Luisa, ${ }^{1}$ and Corsi Fabio ${ }^{1,2}$ \\ ${ }^{1}$ Department of Biomedical and Clinical Sciences "L. Sacco", University of Milan, Via G. B. Grassi 74, 20157 Milan, Italy \\ ${ }^{2}$ Surgery Division, "Luigi Sacco” Hospital, Via G. B. Grassi 74, 20157 Milan, Italy \\ Correspondence should be addressed to Corsi Fabio; fabio.corsi@unimi.it
}

Received 30 March 2016; Accepted 22 May 2016

Academic Editor: Michele Laus

Copyright (C) 2016 Truffi Marta et al. This is an open access article distributed under the Creative Commons Attribution License, which permits unrestricted use, distribution, and reproduction in any medium, provided the original work is properly cited.

\begin{abstract}
Metastatic breast cancer represents a diagnostic and therapeutic challenge due to tumor heterogeneity and to various physiological barriers that hinder drug delivery to the metastatic sites. To overcome these limitations, nanoformulated drugs have been developed and tested in preclinical studies, and few of them have been successfully translated into clinical practice. In particular, liposomal anthracyclines and nanoformulated albumin-bound paclitaxel have revealed an improved therapeutic index when compared to conventional chemotherapy, with significant reduction of drugs toxicity. Several strategies for nanoparticles engineering have more recently been explored to increase selectivity for tumor cells and to reach poorly accessible metastatic districts. Targeted nanoparticles, directed toward tumor markers and tissue-specific metastases, may provide effective devices in case of lowvascularized and small-sized metastases, thus paving the way for a real change in the natural history of metastatic disease. A number of targets have been identified and exploited for surface functionalization of different types of nanoparticles, which are currently undergoing preclinical studies. The aim of this review is to provide an overview of current nanotechnology applied to metastatic breast cancer diagnosis and treatment. Promising results encourage an upcoming translation of this research into clinical practice for an effective management of the disease in the near future.
\end{abstract}

\section{Introduction}

Metastatic breast cancer (MBC) is a major cause of cancerrelated death among women in developed countries [1]. In recent years, some distinct biological portraits of breast cancer, characterized by different biomolecular profiles, behaviours, and clinical courses, have been described, and breast cancer has been defined as a heterogeneous disease $[2,3]$. In this scenario, targeted therapy toward specific cellular receptors has gained a central role in breast cancer management, showing strongly improved clinical outcomes compared to older chemotherapy regimens. A paradigm of this concept was provided by the introduction of tamoxifen as adjuvant therapy for hormone receptors-positive breast tumors, which has led to a fall in cancer recurrence [4]. More recently, while some chemotherapy regimens have failed to increase patients survival, the advent of the antibody trastuzumab has significantly improved the overall survival for human epidermal growth factor receptor 2- (HER2-) positive $\mathrm{MBC}$, a highly aggressive cancer prone to brain metastases [5, 6]. However, onset of resistance to trastuzumab is not uncommon, and it leads to higher risk of local or distant recurrence and poor survival if therapy is not switched to other anti-HER2 strategies [6]. Moreover, HER2-positive cancers account for only $20-25 \%$ of breast malignancies, while other particularly aggressive subsets still represent an unsolved clinical challenge [7]. As an example, triplenegative breast cancer is a particular subset of cancer highly prone to local recurrence and distant metastases, characterized by the lack of expression of the estrogen receptor, progesterone receptor, and HER2, and no targeted therapy is currently available. Beyond targeted therapy, conventional 
chemotherapy for $\mathrm{MBC}$ is generally based on anthracyclines and taxanes. Both these drugs have excellent cytotoxic activity. As a first-line single-agent chemotherapy, doxorubicin leads to response rates of $35-50 \%$ and reduces the risk of death by $22 \%$ with an increased median survival up to 18 months, when compared with nonanthracycline regimens $[8,9]$. More recently, taxanes have been introduced in clinical practice with a substantial benefit in overall survival, time to progression, and cancer response [10]. However, despite their significant antitumor activity, doxorubicin and taxanes are associated with a number of severe side effects due to their toxicity profile. The most dreaded toxicity associated with doxorubicin is cumulative cardiotoxicity, which may lead to irreversible cardiomyopathy and subsequent congestive heart failure; taxanes induce significant neurotoxicity [10,11]. These side effects are dose-dependent and mostly related to the lack of specificity of these drugs, which also affect healthy tissues. Severe toxicity slows down the therapeutic index of these therapies and makes the dose reduction or even the discontinuation of the chemotherapy necessary, with subsequent decreased efficacy. Therefore, MBC remains a largely incurable disease, and novel drug formulations able to achieve specific tumor delivery and to overcome major chemotherapy limitations are required. Moreover, various common sites of breast cancer metastases, such as the brain, are poorly accessible sanctuaries for cancer cells and require smart and innovative drug delivery systems [12]. In this context, nanotechnology represents a promising field of research, since nanoformulation of drugs may improve their bioavailability and allow the selective targeting of tumor cells, with subsequent increased efficacy and lower toxicity. Breast cancer management is the medical field with the most extensive clinical use and the most abundant preclinical research in nanotechnology, and more than 150 clinical trials are currently investigating the efficacy of nanoformulated chemotherapy [13]. The aim of this review is to provide a state of the art in nanotechnology applied to MBC treatment, from nanoformulated drugs already used in clinical practice to novel functionalized nanoparticles for targeted therapy and diagnosis. Ongoing preclinical and clinical studies are expected to provide a significant contribution to the development of new approaches for the management of MBC.

\section{Nanotechnological Approaches for MBC Treatment}

2.1. Nano-Drug-Delivery Systems: Functional and Structural Features. In the last decades, nanotechnology has made many efforts to design innovative and more effective drug delivery systems (DDS) in order to improve the pharmacological treatment of MBC. The main goal in DDS engineering is the achievement of an optimal therapeutic index, which is the combined result of drug release and efficacy, biocompatibility, and stability of the nanodevice and physiological features of the target tissue/organ [62]. The carrier should be nontoxic and easily produced on industrial scale to favour clinical translation [63]. Moreover, it is crucial that the nanodrug displays the highest specificity toward cancer cells in order to increase the delivery of active drug to the tumor while avoiding side effects [64]. The amount of drug molecules loaded in the carrier needs to be adjusted and optimized in order to balance drug dosage and compatible DDS concentration per administration [62]. Generally, nanoparticles larger than $100 \mathrm{~nm}$ are easily recognized and cleared by the reticuloendothelial system; therefore, nanocarrier size refining is essential to provide stability in the bloodstream and proper pharmacokinetics upon parenteral administration $[65,66]$. In the last twenty years, several nanoparticle platforms have been developed in order to improve pharmacokinetics and toxicity of anticancer drugs [67]. Among the first ones, we find liposomes, which are vesicles with a cave sphere structure constituted by single or multiple bilayers of natural or synthetic lipids surrounding an aqueous core. Liposomes are highly biocompatible and biodegradable, and their size can range from few tens of nanometers to micrometers. Since they are able to encapsulate hydrophilic molecules within the aqueous core and hydrophobic agents within their lamellae, liposomes are considered an excellent therapeutic carrier. Another kind of highly used DDS is the albumin-bound nanoparticle (Nab), which exploits the capability of albumin to bind and transport hydrophobic molecules. Moreover, albumin is able to recognize the glycoprotein receptor gp60 and mediate the endothelial transcytosis of $\mathrm{Nab}[68,69]$. Other extensively studied nanodevices for drug delivery include dendrimers, protein-based nanocages, and polymeric and metal nanoparticles. Dendrimers are regularly branched macromolecules produced starting from synthetic or natural elements such as amino acids, sugars, or nucleotides; they can be easily modified or conjugated with therapeutics and loaded with drugs using the cavities of their structure $[35,70]$. Proteinbased nanocages are biological nanoparticles characterized by easy catabolism, minimal toxicity, and immunogenicity. They are constituted by self-assembling protein subunits generally arranged in a hollow structure, which can be chemically or genetically engineered to insert pharmaceuticals and/or probes or to shape various and ordered functionalities, thus enabling the realization of multifunctional nanoparticles. The uniform cage of these nanoparticles allows the precise control of the amount of encapsulated drugs/probes, which is crucial in defining drug dosage. Moreover, their stability in physiological environment avoids macromolecular aggregation, thus increasing circulation time, protecting the cargo molecule from degradation, and improving bioavailability $[71,72]$. Polymeric nanoparticles are made of biocompatible and biodegradable polymers consisting of two or more chains with different hydrophobicity. Through a self-assembling process, these chains form a core-shell structure that reduces the interactions of the hydrophobic blocks with the aqueous environment. These nanoparticles show high loading capacity and are well suited for delivery of hydrophilic or hydrophobic small drugs or macromolecules [36, 73]. Finally, inert metals, such as gold, iron, and titanium, have been used to generate multivalent and theranostic nanosystems, which couple in a single agent both cancer therapy and diagnostics. Indeed, thanks to their physicochemical properties, some inorganic nanoparticles can address other biomedical applications besides the drug delivery, such as the photothermal 
therapy and the tumor imaging. They have been classified as being inert and biocompatible; however, metal nanoparticles retained in the organism after administration may potentially cause toxicity [67]. All nano-DDS developed for MBC management are described below and subdivided according to their use in clinical practice or preclinical research. A complete overview is provided in Table 1 .

\subsection{Nanoparticles in Clinical Use: From Liposomal Anthra-} cyclines to Nanoformulated Paclitaxel. Among the first nanoparticles applied in medicine as drug delivery systems, we find three anthracycline-based nanoformulations: liposomal daunorubicin (DaunoXome), nonpegylated liposomal doxorubicin (Myocet), and pegylated liposomal doxorubicin (Doxil in USA and Caelyx in other countries). DaunoXome is composed of daunorubicin entrapped in a $45 \mathrm{~nm}$ unilamellar vesicle of distearoyl phosphatidylcholine (DSPC) and cholesterol, with a half-life of 4.4 hours [27]. While liposomal daunorubicin is widely approved for the firstline treatment of AIDS-related Kaposi sarcoma, only scarce literature reports on its role in MBC. In a phase I study, DaunoXome has been administered to 16 breast cancer patients with a planned dose escalation from 80 to $180 \mathrm{mg} / \mathrm{m}^{2}$. Mild cardiotoxicity was observed in 3 patients only, but acute dose-limiting toxicity occurred as febrile neutropenia at $120 \mathrm{mg} / \mathrm{m}^{2}$. Moreover, only 2 patients showed objective responses to treatment [27]. Therefore, further analyses are required to determine the safety and efficacy of this nanoformulation. Myocet is composed of doxorubicin loaded into a $180 \mathrm{~nm}$ vesicle of DSPC and cholesterol, with a half-life of 2-3 hours [74]. This nanoformulation is approved in combination with cyclophosphamide for the first-line treatment of $\mathrm{MBC}$ in Canada and Europe. Various randomized controlled trials have investigated Myocet efficacy and toxicity profile compared to conventional doxorubicin. Two phase III studies have compared nonpegylated liposomal doxorubicin versus doxorubicin as a first-line treatment: no differences were observed in anticancer efficacy and median survival, but a significant reduction of cardiotoxicity was evident with liposomal doxorubicin $[18,19]$. Interestingly, nonpegylated liposomal doxorubicin was also compared to epirubicin, a well-known anthracycline analogue with different cardiotoxicity compared to doxorubicin. A phase III randomized trial showed similarly low cardiotoxicity rates, but Myocet showed increased efficacy compared to epirubicin [20]. A metaanalysis performed by the Cochrane Database of Systematic Reviews on the previously described trials confirmed a significant lower rate of cardiotoxicity with Myocet, and also significant reduction in grade 4 neutropenia, grade $>3$ nausea/vomiting, and grade $>3$ diarrhoea was observed [75]. These data strongly suggest that liposomal nanoformulation increases the therapeutic index of anthracyclines mainly by improving the toxicity profile, although keeping substantially comparable efficacy. The reason for decreased toxicity is that intravenously injected liposomes hamper drug exit from the circulation into healthy tissues, while they only extravasate at cancer sites where endothelial capillary junctions are leaky, thus accumulating specifically into the tumor [76]. Doxil is a $100 \mathrm{~nm}$ liposomal doxorubicin coated with protective hydrophilic surface-bound methoxypolyethylene glycol [14]. Pegylation protects liposomes from uptake by the mononuclear phagocyte system, thus modifying drug pharmacokinetics and prolonging liposomes' blood circulation time. Doxil half-life is higher than that of other anthracyclinebased nanoformulations (55 hours), and its biodistribution also differs from that of conventional doxorubicin, as shown by higher tumor concentration at lower dosage [14, 74]. Clinical trials have demonstrated again a reduced risk of cardiotoxicity but no changes in overall survival and response rates; moreover, Doxil led to a $48 \%$ incidence of hand-foot syndrome, a painful erythrodysesthesia that occurs on hand palms and feet soles $[15,16]$.

Beyond anthracyclines, taxanes (paclitaxel and docetaxel) traditionally represent another mainstay of $\mathrm{MBC}$ treatment, with well-established antitumor efficacy [21]. Since they are highly hydrophobic molecules, they are commercialized in combination with solvents (Cremophor EL and ethanol) that, while ensuring drugs solubility, are associated with hypersensitivity reactions; importantly, taxanes are associated with neurotoxicity and enhanced axonal degeneration [77]. To overcome these limitations, a $130 \mathrm{~nm}$ sized nanoformulated albumin-bound paclitaxel (Nab-paclitaxel, Abraxane) has been developed and subsequently approved by FDA, being nowadays in clinical use for various solid tumors, including MBC. After injection, Nab-paclitaxel particles benefit from albumin-mediated enhanced active transport to accumulate into the tumor [22]. A large number of randomized controlled trials have shown that Nab-paclitaxel could be administered at a higher dose than conventional paclitaxel and without any steroid premedication, which are normally used before infusion of taxanes. Moreover, a lower rate of grade 4 neutropenia was observed with Nab-paclitaxel, allowing a better therapy adherence with improved disease control and clinical outcomes $[23,24]$. Due to these significant improvements in breast cancer therapy, a number of randomized trials are currently investigating Nab-paclitaxel in association with biologic agents used in the metastatic setting with encouraging results in terms of reduced neurotoxicity and higher overall response rates, although controversial findings were observed in progression-free and overall survivals [21, $25,26]$. Certainly, further large randomized trials are required to better clarify Nab-paclitaxel activity and optimal dosages in different subtypes of aggressive MBC, but Nab-paclitaxel has anticipated what we can expect from nanotechnology introduction in clinical practice, showing global improvements in both outcome and quality of life of MBC patients, allowing shorter infusion time, avoidance of steroid premedication, and potentially higher administered dosages [21, 22]. Other forms of nanoformulated paclitaxel are in phase II/III trials [78]. A cationic liposomal paclitaxel (EndoTAG) has been designed to enhance binding to negatively charged endothelial cells in tumor blood vessels, with an increased clinical benefit rate for cationic liposomal paclitaxel plus paclitaxel, although a slight increase in grades 3-4 neutropenia was observed [28]. Another nanoformulated taxane is polymeric-micellar paclitaxel (Genexol-PM), a compound of $20-50 \mathrm{~nm}$ diameter with excellent water solubility and stability. A phase II trial performed on $41 \mathrm{MBC}$ s treated with 


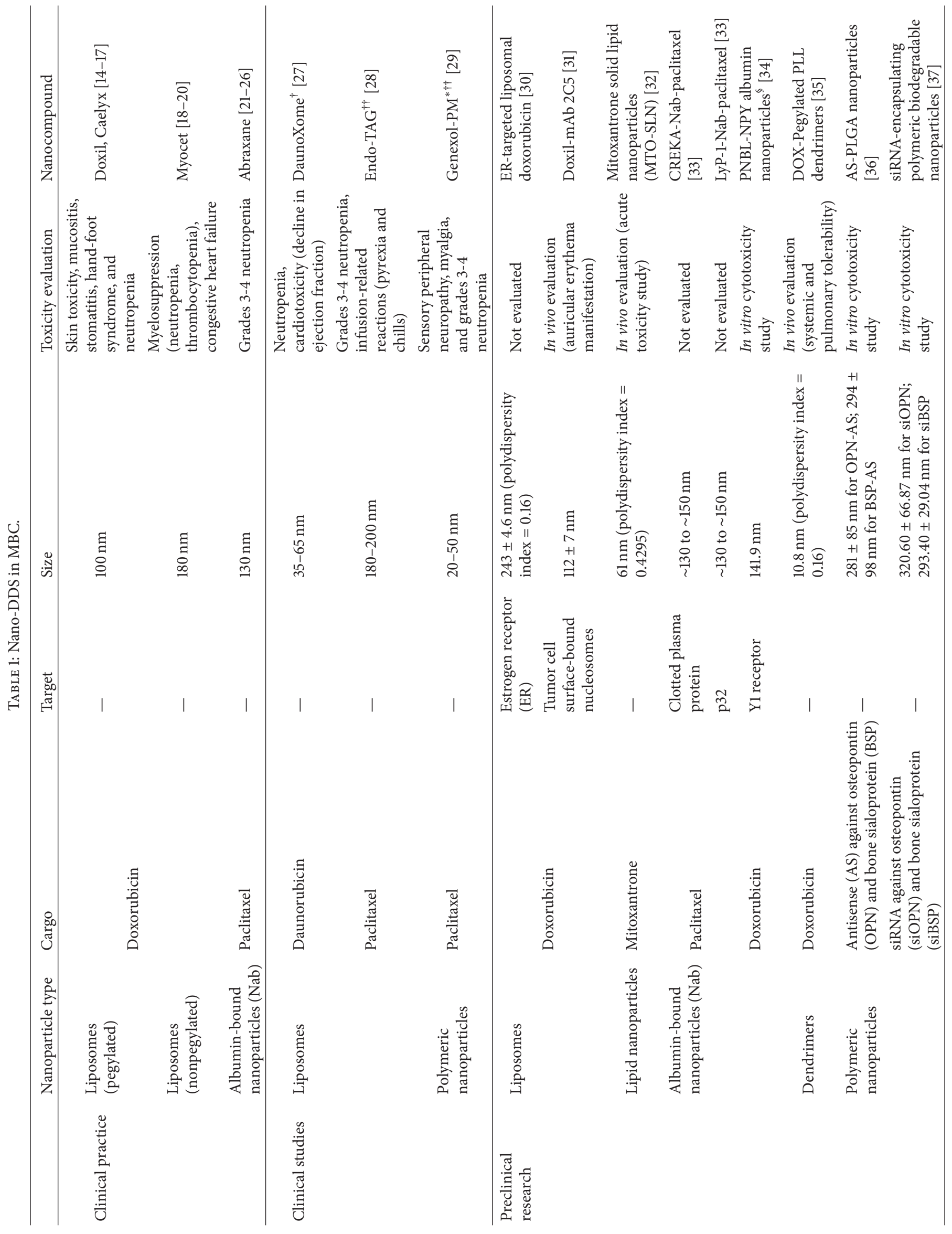




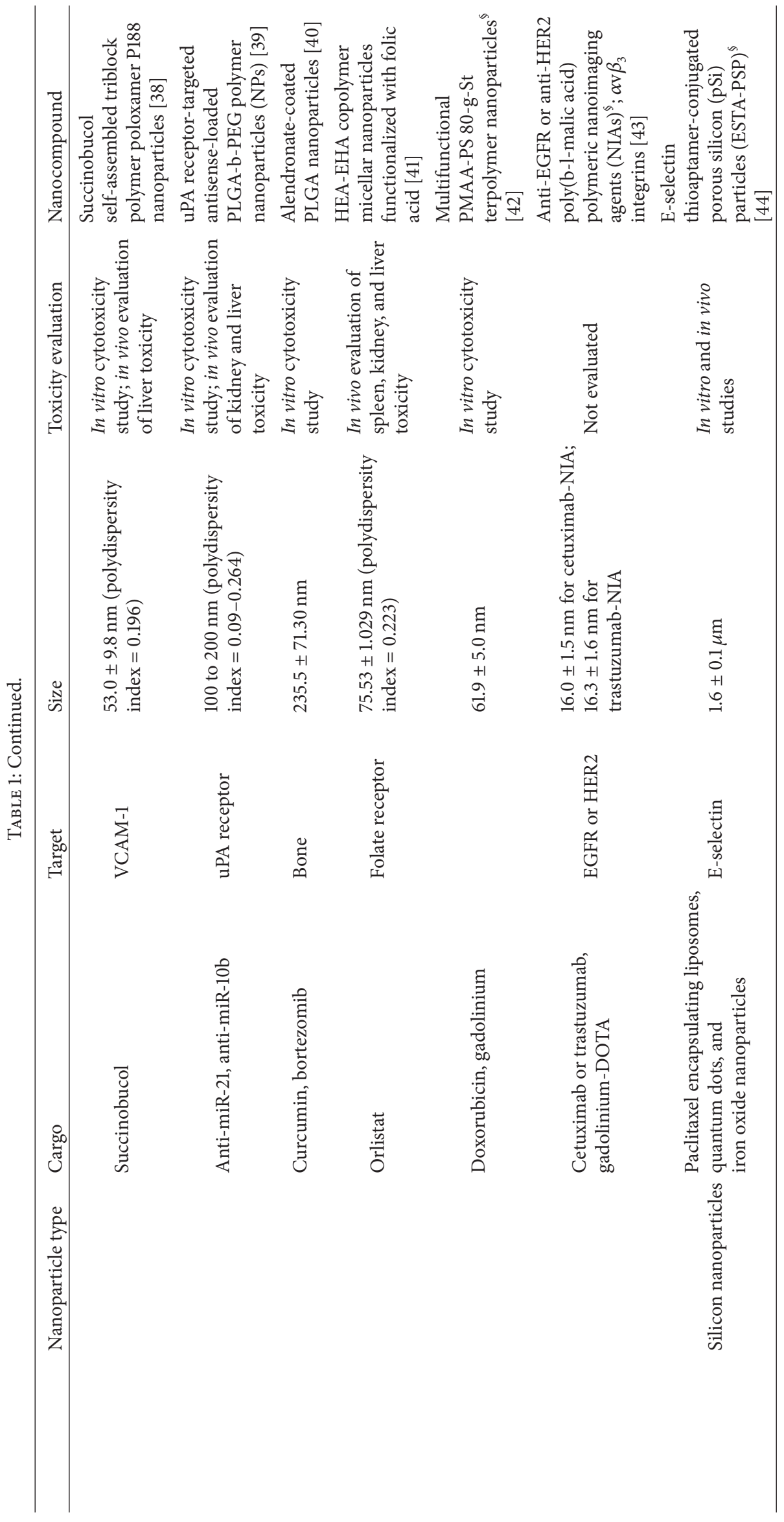




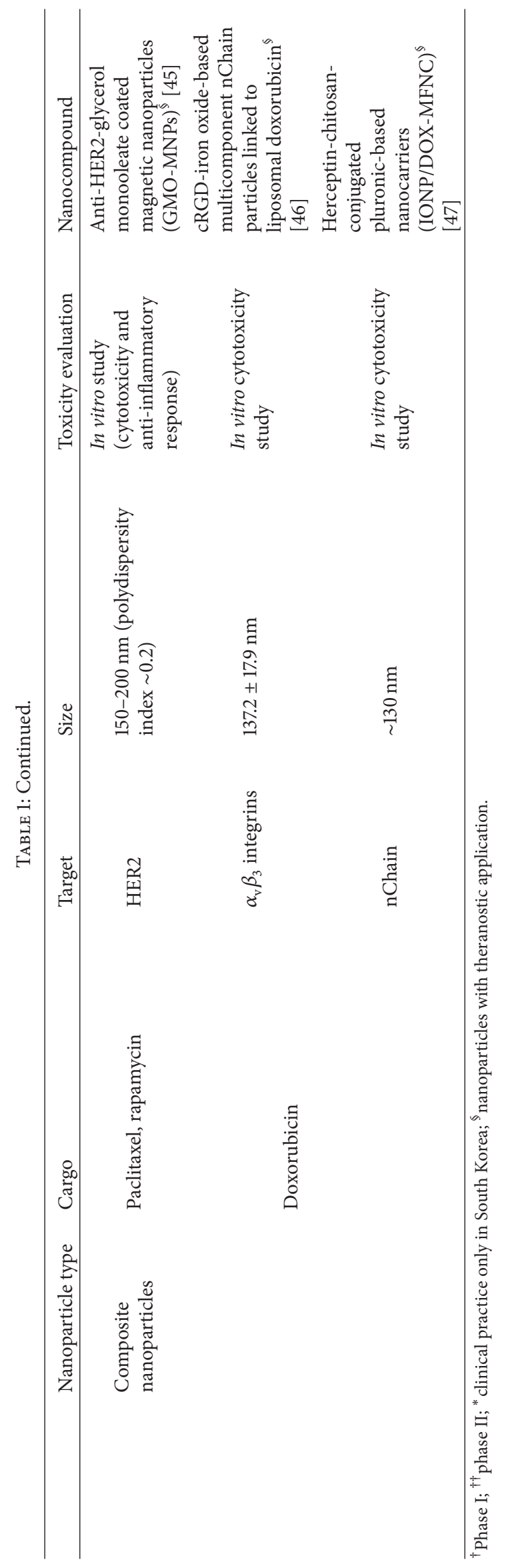


polymeric-micellar paclitaxel as single agent has revealed an objective response rate significantly higher if compared with that obtained at the same dosage with Nab-paclitaxel [29]. Polymeric-micellar paclitaxel is approved in South Korea for use in MBC [78].

\section{A New Outlook from Preclinical Studies: The Active Nanotargeting of MBC}

3.1. Why and How to Target Tumor Cells? Nanotechnological strategies have consistently improved the therapeutic index of anthracyclines and taxanes, mainly by ameliorating their toxicity profiles. However, improvements in clinical outcomes are controversial, and no substantial changes in overall survival have been observed compared to conventional chemotherapy in most cases. This piece of data probably reflects the fact that nanoparticles often accumulate into the tumor mass by enhanced permeability and retention (EPR) effect, while hardly entering the tumor cells. The EPR effect allows proteins larger than $30 \mathrm{kDa}$ to passively extravasate from the blood vessels into the tumor interstitial space, thanks to the increased vascular permeability in correspondence with the tumor endothelium. Indeed, during tumor growth, neoangiogenesis is promoted in order to satisfy the increasing oxygen demand and, at the same time, the endothelium of the new blood vessels is tortuous, poorly differentiated, and leaky, thus allowing molecules extravasation. Moreover, solid tumors lack functional lymphatics and are unable to eliminate extravasated nanomaterials, thus contributing to increasing nanoparticles accumulation and prolonged retention within the tumor $[79,80]$. Therefore, the EPR effect has become one of the most exploited biological phenomena for nanotherapeutic strategies. Nonetheless, it is a complex process depending on many factors such as tumor type, tumor microenvironment, and nanoparticle properties, and it does not ensure the intracellular delivery of the drug [79]. Certainly, the passive diffusion of nanoformulated drugs through leaky tumor vasculature may have a prominent role for the treatment of primary tumors with more than $100 \mathrm{~mm}^{3}$ in volume; however, it remains less effective in a metastatic setting. Metastases are only poorly accessible to molecular or nanoparticle agents because of their small size, high dispersion to organs, and low vascularization if compared to primary tumors [12, 81]. As a consequence, the EPR effect is not sufficient for proper drug nanodelivery to these sites, and the development of effective therapies toward metastases still remains a main challenge in cancer treatment. In this context, nanotechnologists have developed new systems meant to specifically target cancer cells, thus enabling a more effective recognition of the lesion, prolonged persistence at tumor site, and an increasing diagnostic and therapeutic efficacy. This strategy, called "active targeting," involves the conjugation on the nanoparticle surface of targeting moieties, like antibodies or peptides, which specifically bind to tumor receptors or biomarkers and subsequently trigger nanoparticle internalization [80]. Targeted nanoparticles may benefit from the EPR effect but, thanks to the specific binding to the target, also improve tumor delivery of therapeutics and uptake by cancer cells [67]. Liposomal anthracyclines such as Doxil have been functionalized with anticancer antibodies, and preclinical studies have demonstrated improved therapeutic index and reduced side effects when compared to original Doxil [31]. Nab-paclitaxel has also been functionalized with the peptide Lyp-1, which recognizes tumor lymphatics: this study has demonstrated an increased tumor growth inhibition after treatment with targeted Nab-paclitaxel in comparison with untargeted Nabpaclitaxel [33]. By delivering pharmacological agents more selectively into tumor cells, targeted nanoparticles modify the biodistribution of therapeutic drugs and guide them away from healthy tissues, further improving the efficacytoxicity ratio when compared to untargeted nanocompounds [82]. Successful active targeting to MBC basically relies on a thorough understanding of the complex processes involved in tumor growth, invasion, and evolution toward metastasis and metastasis-specific features. Certainly, a major concern is the need of specific and reliable targets, with blown and persistent overexpression in cancer tissues. Recently, the neuropeptide Y1 receptor has been recognized as a novel target for breast cancer cells, since healthy breast tissue overexpresses only Y2 receptors. Therefore, albumin nanoparticles loaded with doxorubicin and functionalized with PNBL-NPY, a ligand of Y1 receptors, have been successfully used to properly address the nanodrug to breast cancer cells [34].

3.2. Targeting the Metastatic Primary Tumor. In the past few decades, significant progress has been made in understanding the genetics and the molecular principles contributing to malignant transformation and tumorigenesis. Gene expression profiling has been used to classify tumors, based on molecular fingerprints that correlate with histological subtypes and may predict the clinical outcome [83]. In particular specific molecules or pathways, either overexpressed or dysregulated in different types of $\mathrm{MBC}$, have been identified and draw interest for targeted applications. HER2 is one of the most commonly targeted molecules in MBC therapy, since it is overexpressed in a large subset of aggressive breast cancers. Antibodies that recognize HER2, such as trastuzumab (Herceptin), represent a gold standard for the molecularly targeted therapy [84]. In this context, a variety of nanotherapeutic strategies based on the conjugation of Herceptin or fragments of it to nanoparticles surface have been explored for targeted delivery to HER2-positive breast cancer and have led to enhanced localization and treatment of tumors both in vitro and in vivo [45, 85-89]. Recently, HER2-targeted nanocarriers have been intensely explored to specifically deliver and release doxorubicin in HER2-positive cancer cells, with enhanced anticancer activity compared to nontargeted nanoparticles [47]. Besides HER2, more than two-thirds of breast cancer cells display upregulation of the estrogen receptor, which has therefore been selected as the most important target for hormone therapy of breast cancer [30]. Several drug delivery platforms have been successfully engineered in order to bind other specific receptors that are prone to endocytosis, such as the folate receptor, the transferrin receptor-1 (TfR-1), and the epidermal growth factor receptor (EGFR), thus enabling drugs to enter the 
cytoplasm of cancer cells and to further exert their antitumoral effects [41, 90-92]. Biomolecules resembling receptors ligands or fragments of them have been studied and produced to be exploited in this context. This is the case for the aminoterminal fragment of the urokinase type plasminogen activator (uPA), which has been used as a targeting moiety for conjugation to the surface of iron oxide-based nanoparticle in order to achieve specific recognition of uPA receptorexpressing cancer cells [54]. A novel approach has been attempted with smart polymeric nanoparticles functionalized with uPA and loaded with antisense miRNAs to antagonize miR-21, responsible for apoptosis inhibition, and miR-10b, which induces metastatic features [39]. Interestingly, these nanoparticles have been investigated in murine model of triple-negative breast cancer, which is known to overexpress uPA receptor [93], and have provided significant reduction of tumor growth and metastases at a very low dose $(0.15 \mathrm{mg} / \mathrm{kg})$, thus proving to be a potential novel therapeutic option for MBC.

3.3. Targeting the Tumor Vasculature. In order to overcome drawbacks in reaching tumor cells, various endothelialtargeted nanodevices have been also developed [94]. These nanoparticles either bind and kill the endothelial target, stopping the flow of oxygen and nutrients to the tumor, or are designed to release the drug directly within the vessel, thus allowing it to go deep in the interstitium [95]. Several endothelial adhesion molecules involved in migration, invasion, and metastatic behaviour of cancer cells represent good targets for nanoparticles design. Ligands immobilized on nanoparticles surface to target tumor vasculature are the antibody fragment L19 and some derivatives of the RGD or NGR peptides, which recognize the EBD domain of fibronectin and the integrins $\alpha_{2} \beta_{5}, \alpha_{\mathrm{v}} \beta_{3}$, and $\alpha_{5} \beta_{1}$, respectively [96, 97]. One interesting example is the multicomponent nanodevice developed by Peiris and coworkers, which consists of iron oxide-based nanospheres linked to a doxorubicin-loaded liposome. This device is functionalized on both liposome and iron oxide nanospheres with the cyclic RGD peptide that targets the $\alpha_{\mathrm{v}} \beta_{3}$ integrins and cell adhesion molecules that mediate the metastatic site transition from tumor cell rolling on the endothelium to firm attachment for subsequent extravasation and tissue invasion. The active targeting provides the specific delivery of the nanoconstruct to tumor neovasculature and, together with size, shape, and flexibility of the construct, contributes to increasing the probability of homing to micrometastases [46]. Endothelial-targeted nanodevices have been shown to possess huge potential for improving antitumor efficacy when compared to tumor celltargeted nanomedicines, and this is mostly due to the fact that they can more easily find their final target.

3.4. Targeting Selective Metastatic Sites and Overcoming Barriers to Cancer Reservoirs. Targeting nanomaterials specifically to the metastatic lesions is a major challenge for translation to clinical use. Expression profiling of metastases arising in different tissues has revealed that gene signature from metastases can be different from that of the original neoplasm [98]. As a consequence, some targeted approaches that generate positive effects in primary tumors could be ineffective toward metastases. Moreover, targeting metastases depend on the specific features of different metastatic sites. Based on the identification of metastasis-specific features, strategies for improving drug delivery to metastatic sites have been developed. Different targets and conjugation strategies have been applied with respect to different types of tumor and metastasis location [12]. A major concern for MBC is the higher occurrence of brain metastases, particularly in some specific subsets [99]. Indeed, most chemotherapeutic drugs have not been generally considered an effective option for patients with central nervous system metastases due to the presence of the blood-brain barrier (BBB), which restricts the access of high-molecular weight molecules from the vasculature [100]. Within the BBB, the tight junctions in between brain microvascular endothelial cells and the P-glycoprotein efflux pumps hamper the filtration of therapeutic drugs, preventing their penetration into the brain. Nanotechnology could be successfully exploited to guarantee a proper addressing of anticancer drugs into the central nervous system, and various strategies are being investigated [101]. A multifunctional polymeric nanotheranostic system was designed in 2014 by $\mathrm{Li}$ et al. and tested in a murine model of breast cancer metastatic to the brain. This nanocarrier demonstrated a high permeability to $\mathrm{BBB}$, with subsequent efficient release of doxorubicin into metastatic cancer deposits and proper gadolinium extravasation to enable an accurate imaging of metastases on magnetic resonance (MRI) [42]. Pegylated liposomal doxorubicin also demonstrated pharmacokinetics and efficacy advantage over nonliposomal doxorubicin in the treatment of an in vivo model of breast cancer brain metastases [17]. More recently, novel polymeric nanoparticles have been attached to gadolinium, functionalized toward HER2 or EGFR, and injected into mice bearing both lung and breast cancers metastatic to the brain. Nanoparticles were able to pass through the $\mathrm{BBB}$ and to selectively reach metastases; moreover, when gadolinium was replaced with selective inhibitors of HER2 or EGFR, a significant reduction of brain metastases and a longer survival of mice were observed [43]. Other attempts to reach brain tumors have been carried out by active targeting of the BBB, exploiting the overexpression of TfR-1 or apolipoprotein $\mathrm{E}$ (ApoE) receptors on its surface $[102,103]$; promising results suggest that this approach could be of benefit even in MBC management.

Other intriguing progress in the field corresponds to the targeting of bone metastases, a common and devastating consequence of breast cancer that leads to alteration of osteoblast and osteoclast function, with resulting skeletal destruction [104]. E-selectin-targeted nanoparticles have proven to trigger bone marrow enrichment of therapeutics, thus suggesting a promising bone metastasis-specific device [44]. PLGA nanoparticles loaded with antisense oligonucleotides against osteopontin and bone sialoprotein have been successfully used in a rat model of triple-negative breast cancer to demonstrate a significant decrease in tumor bone metastasis incidence and in metastases size [36]. Similar polymeric-based biodegradable nanoformulation of siRNAs against bonespecific genes revealed significant efficacy against migration of breast cancer cells and osteolysis in a rat model of 
skeletal metastases [37]. In 2012, PLGA nanoparticles for active targeting of $\mathrm{MBC}$ were developed by conjugation with alendronate, a bone seeking molecule able to bind to the bone and reduce bone resorption. Simultaneous encapsulation of two chemotherapeutic agents, curcumin and bortezomib, within these nanoparticles revealed antiosteoclastogenesis ability and significant decrease of tumor progression in an in vivo intraosseous model of bone metastasis [40]. Other biologic targets and pathways under investigation include breast cancer-derived interleukins, cytokines, prostaglandins, integrins, growth factors, and proteases that mediate cancerrelated bone resorption [105].

Another serious problem for breast cancer patients is represented by lymph node metastases. Mitoxantrone solid lipid nanoparticles with mean size of less than $100 \mathrm{~nm}$ were prepared, characterized, and tested by subcutaneous injection in a model of lymph node metastases [32]. Results showed high targeting efficiency of the drug to the lymph nodes, enhanced therapeutic effect of the antitumor agent, and significant reduction in lymph node mean size.

Other researchers have exploited the vascular cell adhesion molecule-1 (VCAM-1) as a therapeutic target against lung metastasis of breast cancer. They have generated hydrophobic interaction-mediated self-assembled nanoparticles with succinobucol, a potent and selective VCAM-1 inhibitor, and have demonstrated improved drug bioavailability and biodistribution upon nanoformulation, together with efficacy in suppressing the lung metastasis of breast cancer in a $4 \mathrm{~T} 1$ breast cancer murine model [38].

3.5. Targeting the Circulating Tumor Cells. Factors that promote the infiltration, survival, and colonization of distant organs are also attractive targets that could be used as prognostic markers for guiding the therapy and/or be themselves good therapeutic targets, to prevent metastases outgrowth. Numerous studies have evaluated the relevance of circulating tumor cells (CTCs) in the peripheral blood as markers of clinical progression in breast cancer and predictive indicators of response to therapy [106, 107]. Detecting CTCs, more than solid organ metastases, seems to offer an effective and reliable prediction of tumor behaviour; however, the lack of specific and robust molecular targets is a prominent limitation for this kind of approach. Galanzha and colleagues have developed gold nanoparticles to detect circulating tumor cells, by targeting CD44: such nanoconstructs have revealed high sensitive and noninvasive detection of cancer cells and their subsequent destruction by ultrasound or hyperthermia, thus preventing metastases formation [55]. More recently, magnetic nanogels based on magnetic nanoparticles and linear thermoresponsive polyglycerol were designed for CTCs capturing. Nanogel surface was decorated with transferrin(Tf-) polyethylene glycol moieties, which provided capture of Tf receptors-positive CTCs [59].

\section{Nanoparticles for MBC Diagnosis}

In the last years, many studies have been directed to the design of new contrast agents allowing easy, reliable, and noninvasive identification of breast cancer [108]. In this context, the development of target-specific nanostructured contrast agents has revealed optimal sensitivity for various imaging techniques, from the optical imaging (fluorescence and bioluminescence detection) to the radionucleotide-based imaging (positron emission tomography (PET) and computed tomography (CT)) or MRI. Magnetic iron oxide nanoparticles are able to enhance the $\mathrm{T}_{2}$-weighted negative contrast of target tissues in MRI and, to date, they represent the prevailing agent for breast cancer detection. Engineering the surface of this nanodevice with specific tumor markers has led to the generation of one of the most studied versatile platforms for site-specific tumor imaging and localization of primary breast cancer by MRI [50, 54, 61]. Nevertheless, the localization of peripheral metastasis still remains a key challenge, and only a limited number of studies have reported on this so far. Here, we describe preclinical nanotechnological research focused on $\mathrm{MBC}$ diagnostics and provide an overview of all developed nanoparticles for MBC imaging in Table 2.

In an interesting study, superparamagnetic iron oxide nanoparticles (SPIONs) have been functionalized with the luteinizing hormone/chorionic gonadotropin (LH/CG) and the luteinizing hormone releasing hormone (LHRH), whose receptors are expressed in $>50 \%$ of breast cancers [51]. It was found that both types of these small $(<50 \mathrm{~nm}$ hydrodynamic diameter) and highly soluble nanoparticles were actively internalized in vitro by the MDA-MB-435S breast cancer cells after binding to their receptors. In vivo investigation on nude mice bearing a MDA-MB-435S interscapular tumor showed a strong accumulation of intravenously injected LHRH-conjugated SPIONs (up to 59\% of injected iron particles) within the primary tumor but also within lung metastases (up to 20\%). MRI of breast cancers dissected after 20-hour exposure to LHRH-conjugated SPIONs showed that these nanoparticles were able to enhance the negative contrast of the xenografts [52]. Multi-CRAZED magnetic anisotropy images of isolated tissues confirmed the great potential of LHRH-conjugated SPION as contrast agent for lung metastases [52]. Kievit and colleagues have designed a SPION coated with a copolymer of chitosan and polyethylene glycol and functionalized with a monoclonal antibody against human HER2/neu [60]. This neu-conjugated nanoparticle (NP-neu) has been shown to be efficiently internalized by neu-expressing mouse mammary carcinoma cells in vitro. The ability of NP-neu to target MBC and to be detected by MRI was assessed in transgenic mice that spontaneously develop HER2/neu-positive tumors: a significant $\mathrm{T}_{2}$ decrease was observed at the tumor site in NP-neu-injected mice as compared to the noninjected animals. Lung, liver, and bone marrow metastases were also detected by histological and cytofluorimetric assays upon NP-neu injection. Positive Prussian blue staining of histological sections indicated the presence of iron nanoparticles in lung and liver metastases. This targeting effect on liver and lung by NP-neu was further confirmed using flow cytometry analysis, which also provided a successful detection of metastatic cancer cells in aspirated bone marrow. Although able to selectively label early stage micrometastases by histology or flow cytometry, these neu-targeted nanoparticles have finally been shown to be useless for MRI, since the micrometastases were beyond 


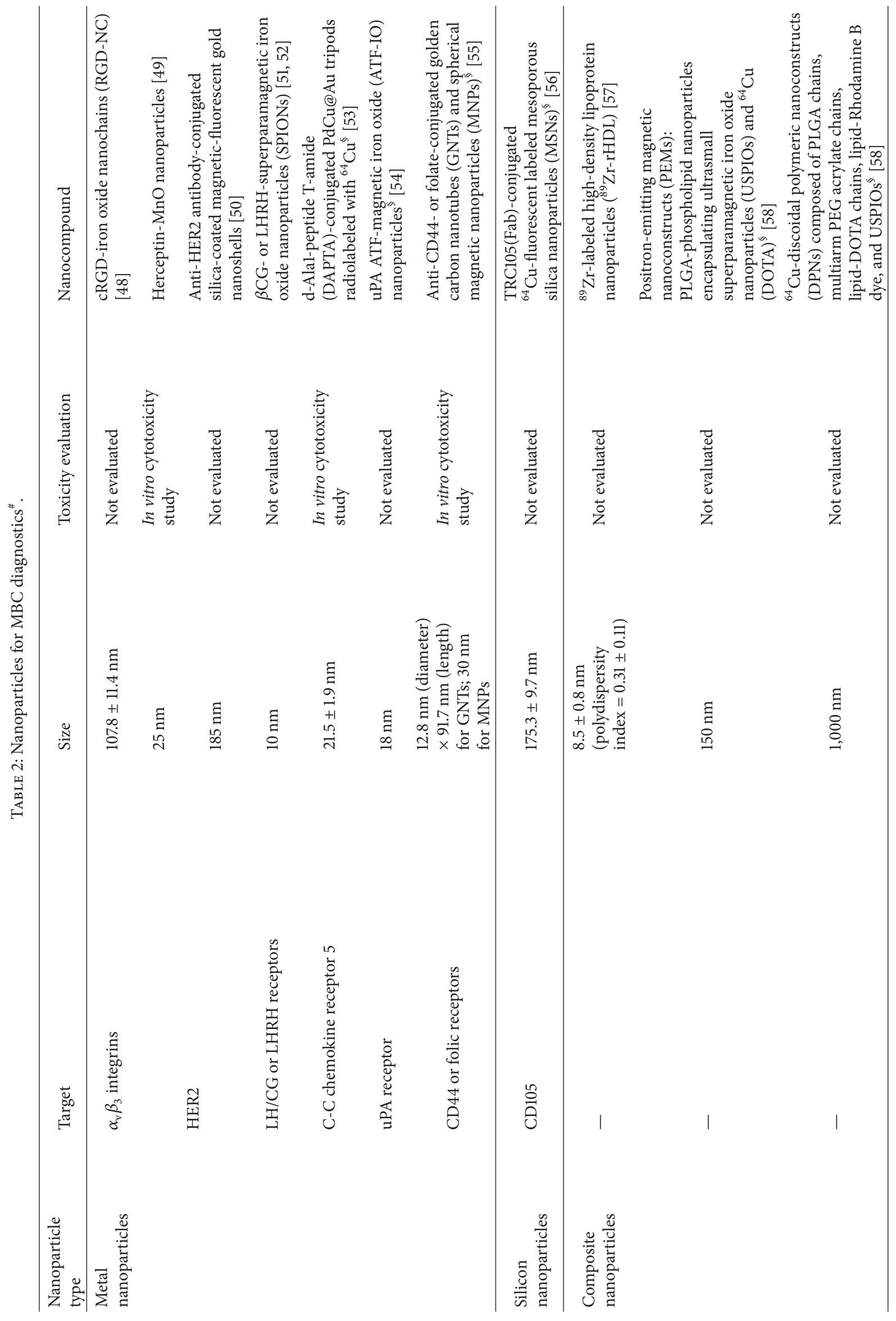




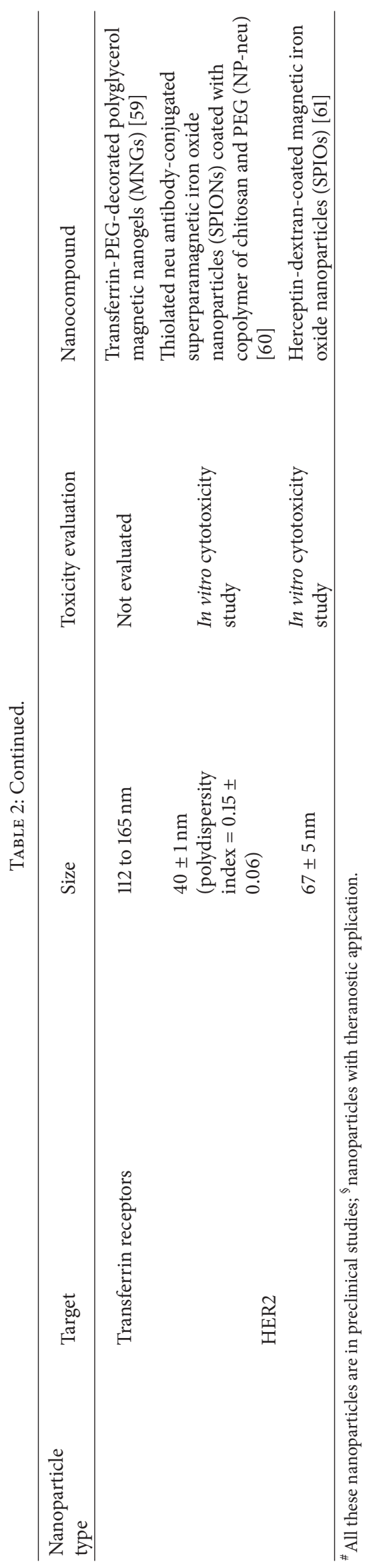


the spatial resolution of a 3-Tesla MRI. In the same year, Pereis and colleagues developed a nanochain (100 nm hydrodynamic size) of four iron oxide nanoparticles functionalized with a cyclic tripeptide arginine-glycine-aspartic acid $c$ (RGD), for the specific targeting of vascular $\alpha_{\mathrm{v}} \beta_{3}$ integrins [48]. Fluorescence Molecular Tomography (FMT) imaging showed that these nanochain-RGD nanoparticles (RGD-NC) were able to actively target primary $4 \mathrm{~T} 1$ mammary tumors in mice within 30 minutes from intravenous injection. Targeting of metastatic lesions by RGD-NC was also observed in vivo and ex vivo in liver, spleen, and lungs. MRI images of the liver in metastatic mice injected with RGD-NC showed a significantly higher negative contrast in this organ when compared to the preinjection contrast or to the postinjection background signal of healthy or uninvolved regions of the metastatic liver. Fluorescence microscopy observations of histological sections showed the microdistribution of RGD$\mathrm{NC}$ in liver and lungs, indicating the colocalization of the nanoparticles with both cancer cells and $\alpha_{v} \beta_{3}$ integrins. Most recently, the addition of iron oxide nanoparticles to HER2targeted doxorubicin nanocarriers allowed for obtaining a promising theranostic agent, suitable for both HER2-positive breast cancer treatment and MRI diagnosis [47].

Although SPION is still the most studied nanoparticlebased contrast agent for MRI detection of breast cancer and its metastases, some limitations in its use are associated with the negative contrast and the magnetic susceptibility artefacts provided by iron oxide. Therefore, some researchers have focused on a new class of contrast agent with positive ( $\mathrm{T}_{1}$-weighted) contrast ability. In this context, $\mathrm{Na}$ and colleagues have developed manganese oxide $(\mathrm{MnO})$ nanoparticles ( $25 \mathrm{~nm}$ diameter) functionalized with the anti-HER2 Herceptin, for MRI detection of breast cancer metastases in mice brain [49]. After intravenous injection of Herceptin$\mathrm{MnO}$ nanoparticles, metastases were significantly enhanced in $\mathrm{T}_{1}$-weighted MRI as a result of the active targeting and the accumulation of nanoparticles into breast cancer cells. Nonfunctionalized $\mathrm{MnO}$ nanoparticles were also observed to reach the brain metastases by passing through the leaky BBB damaged by tumor formation within the brain. However, only functionalized nanoparticles were able to accumulate at the tumor site for an extended time thanks to their interaction with HER2.

Beyond MRI, PET imaging is indicated to complete the staging of $\mathrm{MBC}$, due to its whole-body detection of tumor-related metabolic activity. However, its spatial resolution, its specificity, and sometimes uptake of ${ }^{18} \mathrm{~F}$-fluoro2-deoxy-D-glucose are not optimal [109]. Recently, Pang et al. have demonstrated that $\mathrm{PdCu} @ A u$ nanoparticles radiolabeled with $64_{\mathrm{Cu}}$ and functionalized toward C-C chemokine receptor 5, a newly identified target of triple-negative breast cancer, were able to specifically recognize $4 \mathrm{~T} 1$ mouse breast cancer cells increasing by 2 - to 6 -fold higher recognition compared to nontargeted nanoparticles, thus providing a tool for highly accurate PET imaging and photothermal treatment [53]. Interestingly, Pérez-Medina et al. have developed a ${ }^{89} \mathrm{Zr}$-labeled liposome encapsulating a near-infrared fluorophore for both PET and optical imaging of breast cancer. Intravenous injection of the nanotracer demonstrated its usefulness in bimodal imaging of cancer deposits by simultaneous PET and intraoperative optical imaging, which is an increasing field of interest in surgical oncology [57]. Mesoporous silica nanoparticles have been also investigated as dual-imaging tracer in murine models of metastatic breast cancer for both PET and near-infrared optical imaging with encouraging results regarding the targeting of both lung metastases and tumor vasculature $[56,58]$.

\section{Conclusions}

In MBC management, conventional chemotherapy shows major limitations in achieving a proper treatment. Although important improvements have been obtained with nanoformulated anthracyclines and paclitaxel, an accurate detection and a satisfying treatment of the metastatic disease still remain a key challenge. In this context, the active targeting of malignant cells appears as a promising strategy for a successful theranostic approach in MBC. Specific ligands or antitumor drugs conjugated on the surface of nanoparticles have demonstrated efficient interaction with cancer cells and improved drug delivery to tumor sites, even through physiological barriers. Active targeting nanostrategies are expected to reduce toxicity and chemoresistance and to enhance the anticancer activity. Certainly, further studies are necessary before introducing this new class of nanodrugs in clinical practice. However, active collaboration between nanotechnologists and physicians has provided satisfactory progress and should be strongly encouraged to achieve a clinical revolution for MBC management.

\section{Competing Interests}

The authors declare that there are no competing interests.

\section{References}

[1] C. DeSantis, J. Ma, L. Bryan, and A. Jemal, "Breast cancer statistics, 2013," CA: A Cancer Journal for Clinicians, vol. 64, no. 1, pp. 52-62, 2014.

[2] S. Glück, F. de Snoo, J. Peeters, L. Stork-Sloots, and G. Somlo, "Molecular subtyping of early-stage breast cancer identifies a group of patients who do not benefit from neoadjuvant chemotherapy," Breast Cancer Research and Treatment, vol. 139, no. 3, pp. 759-767, 2013.

[3] Cancer Genome Atlas Network, "Comprehensive molecular portraits of human breast tumours," Nature, vol. 490, no. 7418, pp. 61-70, 2012.

[4] B. Fisher, J. Costantino, C. Redmond et al., "A randomized clinical trial evaluating tamoxifen in the treatment of patients with node-negative breast cancer who have estrogen-receptorpositive tumors," The New England Journal of Medicine, vol. 320, no. 8, pp. 479-484, 1989.

[5] E. M. Olson, J. S. Najita, J. Sohl et al., "Clinical outcomes and treatment practice patterns of patients with HER2-positive metastatic breast cancer in the post-trastuzumab era," The Breast, vol. 22, no. 4, pp. 525-531, 2013. 
[6] D. M. Gagliato, D. L. Jardim, M. S. Marchesi, and G. N. Hortobagyi, "Mechanisms of resistance and sensitivity to antiHER2 therapies in HER2+ breast cancer," Oncotarget, 2016.

[7] J. O'Shaughnessy, "Extending survival with chemotherapy in metastatic breast cancer," Oncologist, vol. 10, supplement 3, pp. 20-29, 2005.

[8] F. J. Esteva, V. Valero, L. Pusztai, L. Boehnke-Michaud, A. U. Buzdar, and G. N. Hortobagyi, "Chemotherapy of metastatic breast cancer: what to expect in 2001 and beyond," Oncologist, vol. 6, no. 2, pp. 133-146, 2001.

[9] R. P. A'Hern, I. E. Smith, and S. R. Ebbs, "Chemotherapy and survival in advanced breast cancer: the inclusion of doxorubicin in Cooper type regimens," British Journal of Cancer, vol. 67, no. 4, pp. 801-805, 1993.

[10] D. Ghersi, M. L. Willson, M. M. K. Chan, J. Simes, E. Donoghue, and N. Wilcken, "Taxane-containing regimens for metastatic breast cancer," Cochrane Database of Systematic Reviews, no. 6 , Article ID CD003366, 2015.

[11] K. Shan, A. M. Lincoff, and J. B. Young, "Anthracycline-induced cardiotoxicity," Annals of Internal Medicine, vol. 125, no. 1, pp. 47-58, 1996.

[12] A. Schroeder, D. A. Heller, M. M. Winslow et al., "Treating metastatic cancer with nanotechnology," Nature Reviews Cancer, vol. 12, no. 1, pp. 39-50, 2012.

[13] T. Tanaka, P. Decuzzi, M. Cristofanilli et al., "Nanotechnology for breast cancer therapy," Biomedical Microdevices, vol. 11, no. 1, pp. 49-63, 2009.

[14] S. T. Duggan, G. M. Keating, G. Ferrandina, J. P. Kesterson, D. Lorusso, and F. Muggia, "Pegylated liposomal doxorubicin: a review of its use in metastatic breast cancer, ovarian cancer, multiple myeloma and AIDS-related Kaposis sarcoma," Drugs, vol. 71, no. 18, pp. 2531-2558, 2011.

[15] M. E. R. O’Brien, N. Wigler, M. Inbar et al., "Reduced cardiotoxicity and comparable efficacy in a phase III trial of pegylated liposomal doxorubicin $\mathrm{HCl}\left(\mathrm{CAELYX}^{\mathrm{TM}} /\right.$ Doxil $\left.^{\circledR}\right)$ versus conventional doxorubicin for first-line treatment of metastatic breast cancer," Annals of Oncology, vol. 15, no. 3, pp. 440-449, 2004.

[16] T. M. Allen, C. Hansen, F. Martin, C. Redemann, and A. YauYoung, "Liposomes containing synthetic lipid derivatives of poly(ethylene glycol) show prolonged circulation half-lives in vivo," Biochimica et Biophysica Acta (BBA)_Biomembranes, vol. 1066, no. 1, pp. 29-36, 1991.

[17] C. K. Anders, B. Adamo, O. Karginova et al., "Pharmacokinetics and efficacy of PEGylated liposomal doxorubicin in an intracranial model of breast cancer," PLOS ONE, vol. 8, no. 5, Article ID e61359, 2013.

[18] L. Harris, G. Batist, R. Belt et al., "Liposome-encapsulated doxorubicin compared with conventional doxorubicin in a randomized multicenter trial as first-line therapy of metastatic breast carcinoma," Cancer, vol. 94, no. 1, pp. 25-36, 2002.

[19] G. Batist, G. Ramakrishnan, C. S. Rao et al., "Reduced cardiotoxicity and preserved antitumor efficacy of liposomeencapsulated doxorubicin and cyclophosphamide compared with conventional doxorubicin and cyclophosphamide in a randomized, multicenter trial of metastatic breast cancer," Journal of Clinical Oncology, vol. 19, no. 5, pp. 1444-1454, 2001.

[20] S. Chan, N. Davidson, E. Juozaityte et al., "Phase III trial of liposomal doxorubicin and cyclophosphamide compared with epirubicin and cyclophosphamide as first-line therapy for metastatic breast cancer," Annals of Oncology, vol. 15, no. 10, pp. 1527-1534, 2004.
[21] C. Megerdichian, Y. Olimpiadi, and S. A. Hurvitz, "NabPaclitaxel in combination with biologically targeted agents for early and metastatic breast cancer," Cancer Treatment Reviews, vol. 40, no. 5, pp. 614-625, 2014.

[22] S. Glück, "nab-Paclitaxel for the treatment of aggressive metastatic breast cancer," Clinical Breast Cancer, vol. 14, no. 4, pp. 221-227, 2014.

[23] W. J. Gradishar, S. Tjulandin, N. Davidson et al., "Phase III trial of nanoparticle albumin-bound paclitaxel compared with polyethylated castor oil-based paclitaxel in women with breast cancer," Journal of Clinical Oncology, vol. 23, no. 31, pp. 77947803, 2005.

[24] W. J. Gradishar, D. Krasnojon, S. Cheporov et al., "Significantly longer progression-free survival with nab-paclitaxel compared with docetaxel as first-line therapy for metastatic breast cancer," Journal of Clinical Oncology, vol. 27, no. 22, pp. 3611-3619, 2009.

[25] C. Lobo, G. Lopes, O. Baez et al., "Final results of a phase II study of nab-paclitaxel, bevacizumab, and gemcitabine as firstline therapy for patients with HER2-negative metastatic breast cancer," Breast Cancer Research and Treatment, vol. 123, no. 2, pp. 427-435, 2010.

[26] H. S. Rugo, W. T. Barry, A. Moreno-Aspitia et al., "Randomized phase III trial of paclitaxel once per week compared with nanoparticle albumin-bound nab-paclitaxel once per week or ixabepilone with bevacizumab as first-line chemotherapy for locally recurrent or metastatic breast cancer: CALGB 40502/NCCTG N063H (Alliance)," Journal of Clinical Oncology, vol. 33, no. 21, pp. 2361-2369, 2015.

[27] K. J. O’Byrne, A. L. Thomas, R. A. Sharma et al., "A phase I dose-escalating study of DaunoXome, liposomal daunorubicin, in metastatic breast cancer," British Journal of Cancer, vol. 87, no. 1, pp. 15-20, 2002.

[28] A. Awada, I. N. Bondarenko, J. Bonneterre et al., "A randomized controlled phase ii trial of a novel composition of paclitaxel embedded into neutral and cationic lipids targeting tumor endothelial cells in advanced triple-negative breast cancer (tnbc)," Annals of Oncology, vol. 25, no. 4, pp. 824-831, 2014.

[29] K. S. Lee, H. C. Chung, S. A. Im et al., "Multicenter phase II trial of Genexol-PM, a Cremophor-free, polymeric micelle formulation of paclitaxel, in patients with metastatic breast cancer," Breast Cancer Research and Treatment, vol. 108, no. 2, pp. 241-250, 2008.

[30] S. Rai, R. Paliwal, and S. P. Vyas, "Doxorubicin encapsulated nanocarriers for targeted delivery to estrogen responsive breast cancer," Journal of Biomedical Nanotechnology, vol. 7, no. 1, pp. 121-122, 2011.

[31] T. A. Elbayoumi and V. P. Torchilin, "Tumor-specific antibodymediated targeted delivery of Doxil ${ }^{\circledR}$ reduces the manifestation of auricular erythema side effect in mice," International Journal of Pharmaceutics, vol. 357, no. 1-2, pp. 272-279, 2008.

[32] B. Lu, S.-B. Xiong, H. Yang, X.-D. Yin, and R.-B. Chao, "Solid lipid nanoparticles of mitoxantrone for local injection against breast cancer and its lymph node metastases," European Journal of Pharmaceutical Sciences, vol. 28, no. 1-2, pp. 86-95, 2006.

[33] P. P. Karmali, V. R. Kotamraju, M. Kastantin et al., "Targeting of albumin-embedded paclitaxel nanoparticles to tumors," Nanomedicine: Nanotechnology, Biology, and Medicine, vol. 5, no. 1, pp. 73-82, 2009.

[34] J. Li, Z. Shen, X. Ma et al., "Neuropeptide Y Y $\mathrm{Y}_{1}$ receptors meditate targeted delivery of anticancer drug with encapsulated nanoparticles to breast cancer cells with high selectivity and its 
potential for breast cancer therapy," ACS Applied Materials and Interfaces, vol. 7, no. 9, pp. 5574-5582, 2015.

[35] L. M. Kaminskas, V. M. McLeod, G. M. Ryan et al., "Pulmonary administration of a doxorubicin-conjugated dendrimer enhances drug exposure to lung metastases and improves cancer therapy," Journal of Controlled Release, vol. 183, no. 1, pp. 18-26, 2014.

[36] V. Elazar, H. Adwan, T. Bäuerle, K. Rohekar, G. Golomb, and M. R. Berger, "Sustained delivery and efficacy of polymeric nanoparticles containing osteopontin and bone sialoprotein antisenses in rats with breast cancer bone metastasis," International Journal of Cancer, vol. 126, no. 7, pp. 1749-1760, 2010.

[37] C. Reufsteck, R. Lifshitz-Shovali, M. Zepp et al., "Silencing of skeletal metastasis-associated genes impairs migration of breast cancer cells and reduces osteolytic bone lesions," Clinical and Experimental Metastasis, vol. 29, no. 5, pp. 441-456, 2012.

[38] H. Cao, Z. Zhang, S. Zhao et al., "Hydrophobic interaction mediating self-assembled nanoparticles of succinobucol suppress lung metastasis of breast cancer by inhibition of VCAM-1 expression," Journal of Controlled Release, vol. 205, pp. 162-171, 2015.

[39] R. Devulapally, N. M. Sekar, T. V. Sekar et al., "Polymer nanoparticles mediated codelivery of AntimiR-10b and AntimiR-21 for achieving triple negative breast cancer therapy," ACS Nano, vol. 9, no. 3, pp. 2290-2302, 2015.

[40] S. I. Thamake, S. L. Raut, Z. Gryczynski, A. P. Ranjan, and J. K. Vishwanatha, "Alendronate coated poly-lactic-co-glycolic acid (PLGA) nanoparticles for active targeting of metastatic breast cancer," Biomaterials, vol. 33, no. 29, pp. 7164-7173, 2012.

[41] R. Paulmurugan, R. Bhethanabotla, K. Mishra et al., "Folate receptor-targeted polymeric micellar nanocarriers for delivery of orlistat as a repurposed drug against triple-negative breast cancer," Molecular Cancer Therapeutics, vol. 15, no. 2, pp. 221231, 2016.

[42] J. Li, P. Cai, A. Shalviri et al., "A multifunctional polymeric nanotheranostic system delivers doxorubicin and imaging agents across the blood-brain barrier targeting brain metastases of breast cancer," ACS Nano, vol. 8, no. 10, pp. 9925-9940, 2014.

[43] R. Patil, A. V. Ljubimov, P. R. Gangalum et al., "MRI virtual biopsy and treatment of brain metastatic tumors with targeted nanobioconjugates: nanoclinic in the brain," ACS Nano, vol. 9, no. 5, pp. 5594-5608, 2015.

[44] A. P. Mann, T. Tanaka, A. Somasunderam, X. Liu, D. G. Gorenstein, and M. Ferrari, "E-selectin-targeted porous silicon particle for nanoparticle delivery to the bone marrow," Advanced Materials, vol. 23, no. 36, pp. H278-H282, 2011.

[45] F. Dilnawaz, A. Singh, C. Mohanty, and S. K. Sahoo, "Dual drug loaded superparamagnetic iron oxide nanoparticles for targeted cancer therapy," Biomaterials, vol. 31, no. 13, pp. 3694-3706, 2010.

[46] P. M. Peiris, R. Toy, A. Abramowski et al., “Treatment of cancer micrometastasis using a multicomponent chain-like nanoparticle," Journal of Controlled Release, vol. 173, no. 1, pp. 51-58, 2014.

[47] W. I. Choi, J. H. Lee, J.-Y. Kim et al., “Targeted antitumor efficacy and imaging via multifunctional nano-carrier conjugated with anti-HER2 trastuzumab," Nanomedicine: Nanotechnology, Biology, and Medicine, vol. 11, no. 2, pp. 359-368, 2015.

[48] P. M. Peiris, R. Toy, E. Doolittle et al., "Imaging metastasis using an integrin-targeting chain-shaped nanoparticle," ACS Nano, vol. 6 , no. 10, pp. 8783-8795, 2012.
[49] H. B. Na, J. H. Lee, K. An et al., "Development of a T1 contrast agent for magnetic resonance imaging using $\mathrm{MnO}$ nanoparticles," Angewandte Chemie-International Edition, vol. 46, no. 28, pp. 5397-5401, 2007.

[50] R. Bardhan, W. Chen, M. Bartels et al., "Tracking of multimodal therapeutic nanocomplexes targeting breast cancer in vivo," Nano Letters, vol. 10, no. 12, pp. 4920-4928, 2010.

[51] C. Leuschner, C. S. S. R. Kumar, W. Hansel, W. Soboyejo, J. Zhou, and J. Hormes, "LHRH-conjugated magnetic iron oxide nanoparticles for detection of breast cancer metastases," Breast Cancer Research and Treatment, vol. 99, no. 2, pp. 163-176, 2006.

[52] J. Meng, J. Fan, G. Galiana et al., "LHRH-functionalized superparamagnetic iron oxide nanoparticles for breast cancer targeting and contrast enhancement in MRI," Materials Science and Engineering C, vol. 29, no. 4, pp. 1467-1479, 2009.

[53] B. Pang, Y. Zhao, H. Luehmann et al., “64 Cu-doped PdCu@Au tripods: a multifunctional nanomaterial for positron emission tomography and image-guided photothermal cancer treatment," ACS Nano, vol. 10, no. 3, pp. 3121-3131, 2016.

[54] L. Yang, X.-H. Peng, Y. A. Wang et al., "Receptor-targeted nanoparticles for in vivo imaging of breast cancer," Clinical Cancer Research, vol. 15, no. 14, pp. 4722-4732, 2009.

[55] E. I. Galanzha, J.-W. Kim, and V. P. Zharov, "Nanotechnologybased molecular photoacoustic and photothermal flow cytometry platform for in-vivo detection and killing of circulating cancer stem cells," Journal of Biophotonics, vol. 2, no. 12, pp. 725735, 2009.

[56] F. Chen, T. R. Nayak, S. Goel et al., "In vivo tumor vasculature targeted PET/NIRF imaging with TRC105(Fab)-conjugated, dual-labeled mesoporous silica nanoparticles," Molecular Pharmaceutics, vol. 11, no. 11, pp. 4007-4014, 2014.

[57] C. Pérez-Medina, J. Tang, D. Abdel-Atti et al., "PET imaging of tumor-associated macrophages with ${ }^{89} \mathrm{Zr}$-labeled high-density lipoprotein nanoparticles," Journal of Nuclear Medicine, vol. 56, no. 8, pp. 1272-1277, 2015.

[58] J. Key, Y.-S. Kim, F. Tatulli et al., "Opportunities for nanotheranosis in lung cancer and pulmonary metastasis," Clinical and Translational Imaging, vol. 2, no. 5, pp. 427-437, 2014.

[59] M. Asadian-Birjand, C. Biglione, J. Bergueiro et al., "Transferrin decorated thermoresponsive nanogels as magnetic trap devices for circulating tumor cells," Macromolecular Rapid Communications, vol. 37, no. 5, pp. 439-445, 2016.

[60] F. M. Kievit, Z. R. Stephen, O. Veiseh et al., "Targeting of primary breast cancers and metastases in a transgenic mouse model using rationally designed multifunctional SPIONs," ACS Nano, vol. 6, no. 3, pp. 2591-2601, 2012.

[61] T.-J. Chen, T.-H. Cheng, C.-Y. Chen et al., "Targeted Herceptindextran iron oxide nanoparticles for noninvasive imaging of HER2/neu receptors using MRI," Journal of Biological Inorganic Chemistry, vol. 14, no. 2, pp. 253-260, 2009.

[62] A. MaHam, Z. Tang, H. Wu, J. Wang, and Y. Lin, "Protein-based nanomedicine platforms for drug delivery," Small, vol. 5, no. 15, pp. 1706-1721, 2009.

[63] R. Duncan, "Polymer conjugates as anticancer nanomedicines," Nature Reviews Cancer, vol. 6, no. 9, pp. 688-701, 2006.

[64] R. Fanciullino, J. Ciccolini, and G. Milano, "Challenges, expectations and limits for nanoparticles-based therapeutics in cancer: a focus on nano-albumin-bound drugs," Critical Reviews in Oncology/Hematology, vol. 88, no. 3, pp. 504-513, 2013.

[65] P. Zarogoulidis, E. Chatzaki, K. Porpodis et al., "Inhaled chemotherapy in lung cancer: future concept of nanomedicine," 
International Journal of Nanomedicine, vol. 7, pp. 1551-1572, 2012.

[66] D. C. Drummond, O. Meyer, K. Hong, D. B. Kirpotin, and D. Papahadjopoulos, "Optimizing liposomes for delivery of chemotherapeutic agents to solid tumors," Pharmacological Reviews, vol. 51, no. 4, pp. 691-743, 1999.

[67] A. Z. Wang, R. Langer, and O. C. Farokhzad, "Nanoparticle delivery of cancer drugs," Annual Review of Medicine, vol. 63, pp. 185-198, 2012.

[68] M. J. Hawkins, P. Soon-Shiong, and N. Desai, "Protein nanoparticles as drug carriers in clinical medicine," Advanced Drug Delivery Reviews, vol. 60, no. 8, pp. 876-885, 2008.

[69] D. A. Yardley, "Nab-Paclitaxel mechanisms of action and delivery," Journal of Controlled Release, vol. 170, no. 3, pp. 365-372, 2013.

[70] P. Kesharwani and A. K. Iyer, "Recent advances in dendrimerbased nanovectors for tumor-targeted drug and gene delivery," Drug Discovery Today, vol. 20, no. 5, pp. 536-547, 2015.

[71] F. Corsi and S. Mazzucchelli, "The potential of protein-based nanocages for imaging and drug delivery," Therapeutic Delivery, vol. 7, no. 3, pp. 149-151, 2016.

[72] M. Truffi, L. Fiandra, L. Sorrentino, M. Monieri, F. Corsi, and S. Mazzucchelli, "Ferritin nanocages: a biological platform for drug delivery, imaging and theranostics in cancer," Pharmacological Research, vol. 107, pp. 57-65, 2016.

[73] G. Kore, A. Kolate, A. Nej, and A. Misra, "Polymeric micelle as multifunctional pharmaceutical carriers," Journal of Nanoscience and Nanotechnology, vol. 14, no. 1, pp. 288-307, 2014.

[74] E. Rivera, "Current status of liposomal anthracycline therapy in metastatic breast cancer," Clinical Breast Cancer, vol. 4, no. 2, pp. S76-S83, 2003.

[75] E. C. van Dalen, E. M. C. Michiels, H. N. Caron, and L. C. M. Kremer, "Different anthracycline derivates for reducing cardiotoxicity in cancer patients," Cochrane Database of Systematic Reviews, no. 4, Article ID CD005006, 2006.

[76] G. Batist, J. Barton, P. Chaikin, C. Swenson, and L. Welles, "Myocet (liposome-encapsulated doxorubicin citrate): a new approach in breast cancer therapy," Expert Opinion on Pharmacotherapy, vol. 3, no. 12, pp. 1739-1751, 2002.

[77] J. Liebmann, J. A. Cook, and J. B. Mitchell, "Cremophor EL, solvent for paclitaxel, and toxicity," The Lancet, vol. 342, no. 8884, p. 1428, 1993.

[78] F. Muggia and D. Kudlowitz, "Novel taxanes," Anti-Cancer Drugs, vol. 25, no. 5, pp. 593-598, 2014.

[79] N. Bertrand, J. Wu, X. Xu, N. Kamaly, and O. C. Farokhzad, "Cancer nanotechnology: the impact of passive and active targeting in the era of modern cancer biology," Advanced Drug Delivery Reviews, vol. 66, pp. 2-25, 2014.

[80] T. Lammers, F. Kiessling, W. E. Hennink, and G. Storm, "Drug targeting to tumors: principles, pitfalls and (pre-) clinical progress," Journal of Controlled Release, vol. 161, no. 2, pp. 175$187,2012$.

[81] T. Lôrincz, J. Tímár, and M. Szendroi, "Alterations of microvascular density in bone metastases of adenocarcinomas," Pathology and Oncology Research, vol. 10, no. 3, pp. 149-153, 2004.

[82] D. Peer, J. M. Karp, S. Hong, O. C. Farokhzad, R. Margalit, and R. Langer, "Nanocarriers as an emerging platform for cancer therapy," Nature Nanotechnology, vol. 2, no. 12, pp. 751-760, 2007.
[83] T. Sørlie, C. M. Perou, R. Tibshirani et al., "Gene expression patterns of breast carcinomas distinguish tumor subclasses with clinical implications," Proceedings of the National Academy of Sciences of the United States of America, vol. 98, no. 19, pp.1086910874, 2001.

[84] D. Slamon, W. Eiermann, N. Robert et al., "Adjuvant trastuzumab in HER2-positive breast cancer," The New England Journal of Medicine, vol. 365, no. 14, pp. 1273-1283, 2011.

[85] M. Colombo, F. Corsi, D. Foschi et al., "HER2 targeting as a two-sided strategy for breast cancer diagnosis and treatment: outlook and recent implications in nanomedical approaches," Pharmacological Research, vol. 62, no. 2, pp. 150-165, 2010.

[86] S. Sommaruga, A. Lombardi, A. Salvadè et al., "Highly efficient production of anti-HER2 scFv antibody variant for targeting breast cancer cells," Applied Microbiology and Biotechnology, vol. 91, no. 3, pp. 613-621, 2011.

[87] L. Fiandra, S. Mazzucchelli, C. De Palma et al., "Assessing the in vivo targeting efficiency of multifunctional nanoconstructs bearing antibody-derived ligands," ACS Nano, vol. 7, no. 7, pp. 6092-6102, 2013.

[88] F. Corsi, L. Fiandra, C. De Palma et al., "HER2 expression in breast cancer cells is downregulated upon active targeting by antibody-engineered multifunctional nanoparticles in mice," ACS Nano, vol. 5, no. 8, pp. 6383-6393, 2011.

[89] S. Mazzucchelli, M. Truffi, L. Fiandra, L. Sorrentino, and F. Corsi, "Targeted approaches for HER2 breast cancer therapy: news from nanomedicine?" World Journal of Pharmacology, vol. 3, no. 4, pp. 72-85, 2014.

[90] Y. Lu and P. S. Low, "Folate-mediated delivery of macromolecular anticancer therapeutic agents," Advanced Drug Delivery Reviews, vol. 54, no. 5, pp. 675-693, 2002.

[91] Z. M. Qian, H. Li, H. Sun, and K. Ho, "Targeted drug delivery via the transferrin receptor-mediated endocytosis pathway," Pharmacological Reviews, vol. 54, no. 4, pp. 561-587, 2002.

[92] M. P. Melancon, W. Lu, Z. Yang et al., "In vitro and in vivo targeting of hollow gold nanoshells directed at epidermal growth factor receptor for photothermal ablation therapy," Molecular Cancer Therapeutics, vol. 7, no. 6, pp. 1730-1739, 2008.

[93] A. M. Le Beau, S. Duriseti, S. T. Murphy et al., "Targeting uPAR with antagonistic recombinant human antibodies in aggressive breast cancer," Cancer Research, vol. 73, no. 7, pp. 2070-2081, 2013.

[94] D. Neri and R. Bicknell, “Tumour vascular targeting," Nature Reviews Cancer, vol. 5, no. 6, pp. 436-446, 2005.

[95] T. Lammers, F. Kiessling, W. E. Hennink, and G. Storm, "Nanotheranostics and image-guided drug delivery: current concepts and future directions," Molecular Pharmaceutics, vol. 7, no. 6, pp. 1899-1912, 2010.

[96] L. Borsi, E. Balza, M. Bestagno et al., "Selective targeting of tumoral vasculature: comparison of different formats of an antibody (119) to the ED-B domain of fibronectin," International Journal of Cancer, vol. 102, no. 1, pp. 75-85, 2002.

[97] A. Mitra, J. Mulholland, A. Nan, E. McNeill, H. Ghandehari, and B. R. Line, "Targeting tumor angiogenic vasculature using polymer-RGD conjugates," Journal of Controlled Release, vol. 102, no. 1, pp. 191-201, 2005.

[98] T. Lorincz, J. Tóth, G. Badalian, J. Tímár, and M. Szendroi, "HER-2/neu genotype of breast cancer may change in bone metastasis," Pathology and Oncology Research, vol. 12, no. 3, pp. 149-152, 2006. 
[99] Z. Gabos, R. Sinha, J. Hanson et al., "Prognostic significance of human epidermal growth factor receptor positivity for the development of brain metastasis after newly diagnosed breast cancer," Journal of Clinical Oncology, vol. 24, no. 36, pp. 56585663, 2006.

[100] J. Hu and S. Kesari, "Strategies for overcoming the blood-brain barrier for the treatment of brain metastases," CNS Oncology, vol. 2, no. 1, pp. 87-98, 2013.

[101] M. Caraglia, G. De Rosa, G. Salzano et al., "Nanotech revolution for the anti-cancer drug delivery through blood-brain-barrier," Current Cancer Drug Targets, vol. 12, no. 3, pp. 186-196, 2012.

[102] C. Cao, X. Wang, Y. Cai et al., "Targeted in vivo imaging of microscopic tumors with ferritin-based nanoprobes across biological barriers," Advanced Materials, vol. 26, no. 16, pp. 2566-2571, 2014.

[103] K. Michaelis, M. M. Hoffmann, S. Dreis et al., "Covalent linkage of apolipoprotein E to albumin nanoparticles strongly enhances drug transport into the brain," Journal of Pharmacology and Experimental Therapeutics, vol. 317, no. 3, pp. 1246-1253, 2006.

[104] G. R. Mundy, "Metastasis to bone: causes, consequences and therapeutic opportunities," Nature Reviews Cancer, vol. 2, no. 8, pp. 584-593, 2002.

[105] P. Clézardin, "Therapeutic targets for bone metastases in breast cancer," Breast Cancer Research, vol. 13, no. 2, article 207, 2011.

[106] N. Bednarz-Knoll, C. Alix-Panabières, and K. Pantel, "Clinical relevance and biology of circulating tumor cells," Breast Cancer Research, vol. 13, no. 6, article 228, 2011.

[107] K. Pantel, C. Alix-Panabières, and S. Riethdorf, "Cancer micrometastases," Nature Reviews Clinical Oncology, vol. 6, no. 6, pp. 339-351, 2009.

[108] M. Ahmed and M. Douek, "The role of magnetic nanoparticles in the localization and treatment of breast cancer," BioMed Research International, vol. 2013, Article ID 281230, 11 pages, 2013.

[109] B. E. Dogan and L. W. Turnbull, "Imaging of triple-negative breast cancer," Annals of Oncology, vol. 23, supplement 6, pp. vi23-vi29, 2012. 

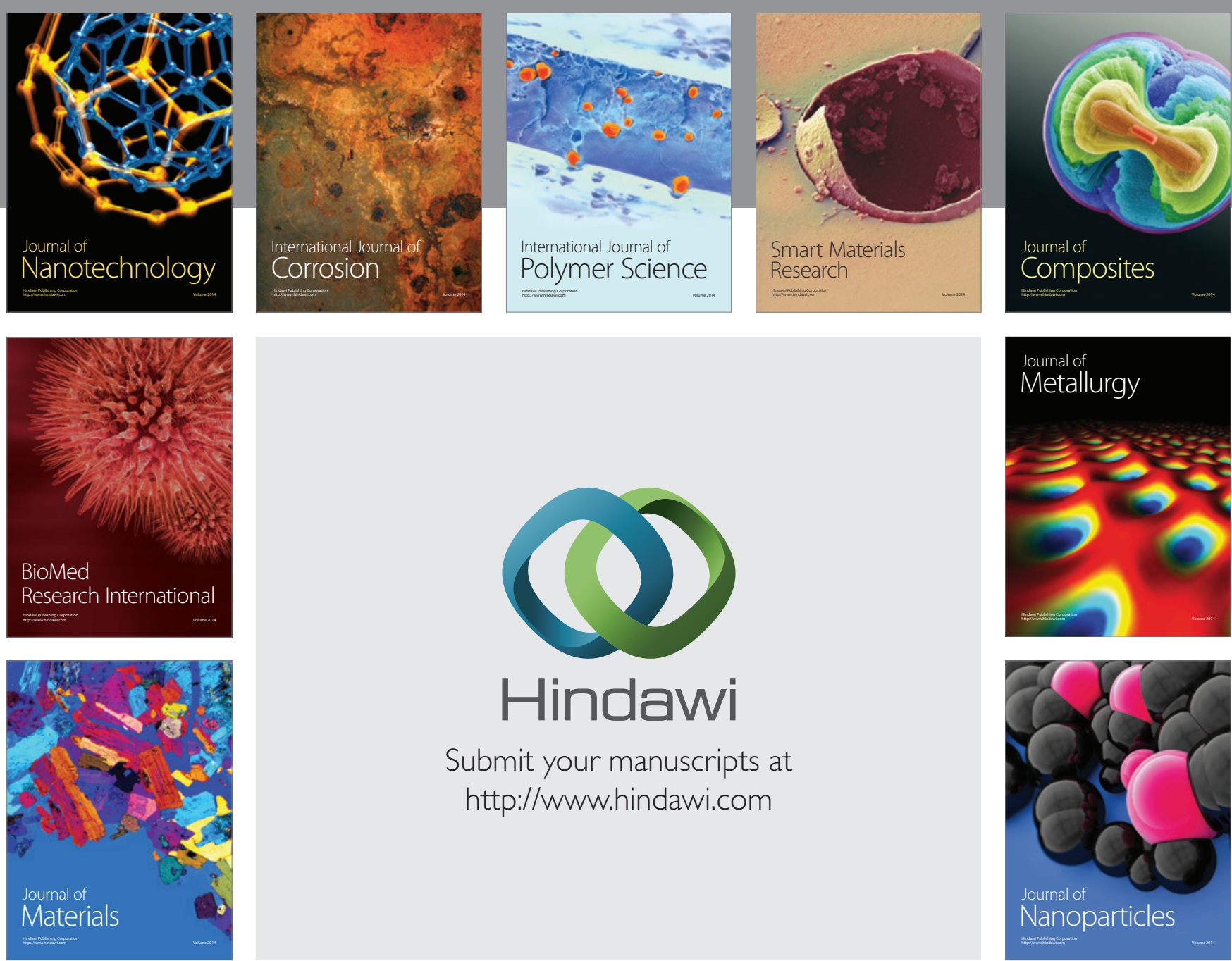

\section{Hindawi}

Submit your manuscripts at

http://www.hindawi.com

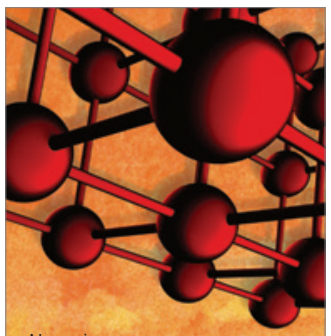

Materials Science and Engineering
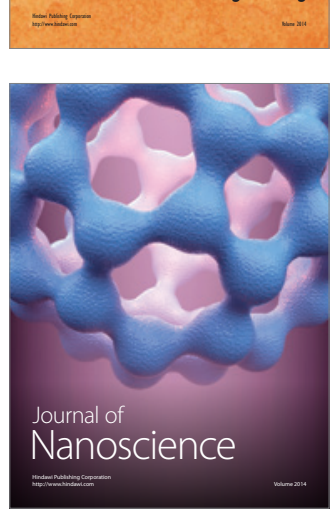
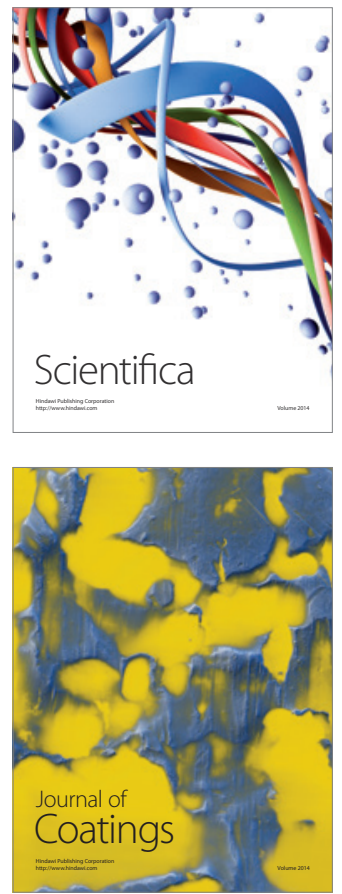
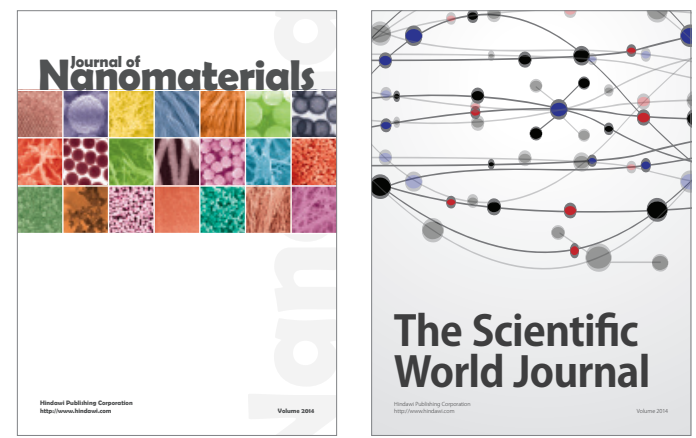

The Scientific World Journal
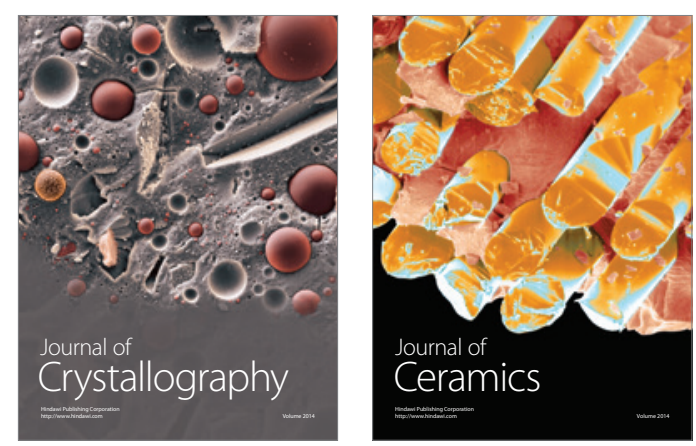
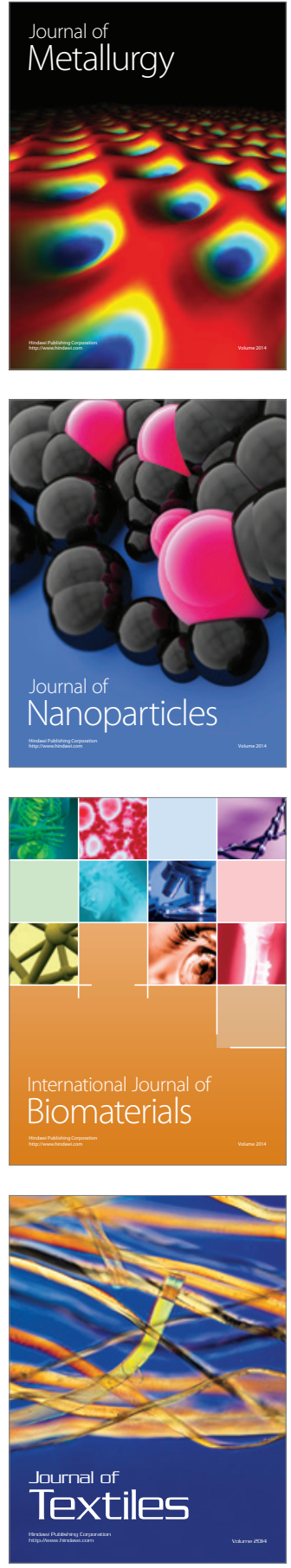